\title{
Améliorer la pratique de la pharmacie au moyen de la technologie : un rêve utopique?
}

\author{
par Jason Howorko
}

A méliorer la sécurité des médicaments, accroître l'efficience des soins de santé et élargir le champ de pratique de la pharmacie ne sont que quelques-uns des avantages de la mise en œuvre de la technologie. En fait, certaines des technologies disponibles aujourd'hui permettraient à la pratique de la pharmacie d'évoluer à pas de géant. Pourtant, de nombreux hôpitaux au Canada continuent de trainer de l'arrière lorsque vient le temps d'adopter ces technologies et systèmes.

Lidée de la distribution de médicaments en doses unitaires a vu le jour au début des années 1960, mais aujourd'hui, 50 ans plus tard, de nombreux établissements canadiens n'ont toujours pas adopté cette méthode de distribution de base. D'après le Rapport 2007-2008 sur les pharmacies hospitalieres canadiennes (www.lillyhospitalsurvey.ca/hpc2/content/2008_ report/20072008F\%20full.pdf), seulement $49 \%$ des répondants au sondage principal (section D) et $62 \%$ des répondants au sondage initial du projet SCPH 2015 (section J) ont déclaré utiliser les systèmes de distribution de doses unitaires pour au moins $90 \%$ des lits de leur établissement.

On ne s'étonnera donc pas que la mise en ouvre des technologies au point d'intervention, y compris la codification à barres, les armoires de distribution automatisée de médicaments, les dossiers pharmaceutiques informatisés, les systèmes informatisés de saisie des ordonnances par les prescripteurs, les outils d'aide à la décision clinique et les dossiers pharmaceutiques partagés entre les hôpitaux et la communauté, semble hors d'atteinte et pouvoir prendre encore plusieurs décennies. Par exemple, comme il en était question dans le Rapport 2007-2008 sur les pharmacies hospitalières canadiennes (section J), seulement $7 \%$ des hôpitaux possèdent des systèmes informatisés de saisie des ordonnances par les prescripteurs et seulement $1 \%$ ont un système de vérification par code-barres des médicaments au chevet du patient.

Si ces technologies ont autant d'avantages, pourquoi leur adoption tarde-t-elle autant au Canada? Les diverses parties prenantes ne se rendent-elles pas compte de l'importance d'utiliser ces technologies pour améliorer le système de soins de santé et accroître la sécurité des patients? Ou le coût serait-il tout simplement trop élevé? Le versement de sommes importantes découlant de procès sera-t-il l'élément déclencheur qui forcera les représentants du système de santé et des gouvernements à

prendre note de ce problème? Ou doit-on poursuivre les efforts de persuasion?

La SCPH continue à faire valoir l'adoption de ces technologies dans les établissements de santé. En voici quelques exemples :

- La SCPH a participé au développement et appuyé le Canadian Pharmaceutical Bar Coding Project: Joint Technical Statement on Pharmaceutical Automated Identification and Product Database Requirements (publié en 2010 par l'Institut pour l'utilisation sécuritaire des médicaments du Canada et l'Institut canadien pour la sécurité des patients; voir www.ismp-canada.org/barcoding/download/CanPharmBarcode_ JointTechnicalStatement.pdf).

- Le projet SCPH 2015 a un objectif complet consacré à l'utilisation des technologies pour améliorer l'utilisation sécuritaire des médicaments (objectif $\mathrm{n}^{\circ}$ 5) (voir www.cshp.ca/ dms/dmsView/1_SCPH-2015---Final-jan\%2710.pdf).

- La SCPH a aussi défendu avec succès les améliorations à la Base de données des produits de santé naturels homologués pour que les outils d'aide à la décision clinique puissent continuer à inclure les produits de santé naturels.

- La SCPH a mené une campagne de valorisation des systèmes de distribution de doses unitaires pour accroître la sécurité des patients.

- La SCPH a des représentants au National e-Pharmacy Task Force et au Comité de direction du Plan directeur pour la pharmacie.

- La SCPH a participé au développement et appuyé le plan de mise en œuvre du Plan directeur pour la pharmacie (voir www.pharmacists.ca/content/about_cpha/whats_happening/ cpha_in_action/pdf/BlueprintImplementationPlan_FR.pdf), qui comprend des produits livrables liés aux technologies de l'information et des communications.

Il est temps de passer à l'action! Notre profession doit veiller à ce que la technologie comme outil critique favorisant la sécurité soit à l'avant-plan. Sinon, nous pourrions bien devoir attendre un autre demi-siècle avant d'en voir la couleur.

[Traduction par l'éditeur]

Jason Howorko, BSP, B. SC., ACPR, est président sortant et agent de liaison externe de la SCPH. 